\title{
Research of Development Status and the Trend of Optical Fiber Communication Technology
}

\author{
Rongrong Wang ${ }^{1, a}$ \\ ${ }^{1}$ Xi'an Shiyou University, Xi'an, Shanxi, 710065 \\ ${ }^{a}$ email
}

Keywords: Optical Fiber, Communication Technology, Development Status and Trend

\begin{abstract}
Not only can be used in optical fiber communications trunk lines of communication, optical fiber can also play a role in power communication control system, industrial monitoring \& control, and now the military has been widely applied, the growing demand for information in various fields based on optical fiber application of communication technology trends are also caused for concern. A complete fiber link is affected by the quality of the fiber itself, but also depends on the fiber optic link field of construction technology and the environment. This paper discusses the main characteristics and development trend of optical fiber communication technology in traditional and mobile internet and we believe this research should be meaningful and the coming research for fiber communication.
\end{abstract}

\section{Introduction}

Currently the explosive growth of data services, more and more crowded road communication, optical communication and therefore become the only way out. Optical fiber communication as a new communications technology, from the outset, showing tremendous advantages, aroused great interest. Compared with the traditional cable transmission, it has a low loss, high communication capacity, immunity to electromagnetic interference, security and good outstanding advantages. Thus, the emergence of optical fiber communication is considered to be a fundamental change in the history of communications. Especially the United States first proposed the "information superhighway" program swept the world, which greatly contributed to its spine - large research and development of optical transmission systems industry. With the construction of B-ISDN to provide broadband transmission of optical fiber communication as the main means of transmission of the twenty-first century communications network will increasingly flourish, showing a strong vitality.

Optical fiber communication theory, the optical modulation can be divided into two categories: direct and indirect modulation. A fiber optic communication complete the whole process, we must first sound source will to be transferred, Electric signals and other images and data into optical signals, and then through the optical fiber transmission to the sink, the sink must be received optical signal light, electricity converted into electrical signals "Thus, compared to fiber optic communication cable to communicate with, there are two main differences: instead of using the optical signal to an electrical signal transmission signal; selection of fiber optic cable as the transmission medium instead.

Optical fiber communication technology has a greater load range than other materials, a wider frequency band than other materials, which means that in the same unit time, optical fiber communication technology can load more information capacity, in the course of optical fiber communication transmission speed and quality is much higher than traditional cable and fiber optic cable, with a high effectiveness and specificity. In the process of optical fiber communication technology applications, which single-wavelength optical fiber communication technology can play a greater degree of fiber wide range of load, bandwidth characteristics, greatly enhance communications transmission capacity, enhance the effect of the underlying DWDM and information transmission quality.

With the gradual reduction in the current number of relay stations, the propagation loss increases. The optical fiber communication technology than the traditional communication media across a 
wider range of distance without repeaters, to a certain extent, reduce the loss in the process of information dissemination, and optical fiber communication technology is still used in the traditional quartz fiber as a medium to communicate spread, less theory if in the future to develop a more economical consumption of non-quartz fiber media, the loss can be reduced, and thus the most economical long-distance transmission of information in the process, reduce the loss of economic costs.

Materials currently used in optical fiber communication technology is an insulator material made of quartz, which is itself the owner of smaller, lesser quality, strong resistance to electromagnetic interference characteristics. The use of optical fiber as a medium of communication can significantly enhance the dissemination of information during the immunity to electromagnetic interference from external electromagnetic nature of information and interference with electronic cable artificially erected can significantly enhance the communication quality of the strong electric field.

In the optical waveguide structure in optical fiber communication technology can be a complete signal transmission, fiber surrounded by opaque foreskin can be absorbed in the process of information transmission missing rays, while the signal source optical fiber transmission mostly in the form of arcs of the transfer, which leads to information during transmission fiber substantially does not leak light, the fiber content can not eavesdrop on the transmission of information, greatly improving the transmission of information in the process of confidentiality.

\section{Status of Optical Fiber Communication Technology}

Ordinary single-mode fiber is the most commonly used fiber. With the development of optical communication systems, optical repeaters distance and single wavelength channel capacity increases, G.652.A optical performance there may be further optimized performance at low attenuation coefficient 1550rim area has not been fully utilized and the optical fiber lowest attenuation coefficient and zero dispersion point not in the same area. Comply ITUTG.654 predetermined cutoff wavelength shifted single-mode optical fiber and in accordance with the provisions of G.653 dispersion-shifted single-mode fiber to achieve such improvements.

China has fully adopted in the trunk (including national backbone, regional and provincial trunk trunk) on the cable, wherein the multi-mode fiber has been eliminated, all with single-mode optical fiber, optical fiber including G.652 and G.655 fiber. G.653 fiber although in our country has been used before, but the future will not develop. G.654 fiber because it is not very substantial increase in the capacity of fiber optic systems, it is not used in our land optical cable. The discrete fiber trunk cable, do not use an optical fiber ribbon. Tight tube layer stranded optical cable trunk and skeletal structure is mainly used outdoors, in these cables, and have been used, has been discontinued.

Access Network cable distance is short, many branches, drop frequently, in order to increase the capacity of the network, usually to increase the fiber count. Especially in the city pipeline, due to the limited pipe diameter, increasing fiber counts while increasing fiber cable set density, reduced cable diameter and weight, it is very important. Access networks G.652 single-mode fiber and G.652.C ordinary low water peak single-mode fiber. Low water peak single-mode optical fiber suitable for dense wavelength division multiplexing, there are a small amount of use in our country.

Indoor fiber optic cable is often required for both transmission of voice, data and video signals. And also may be used for telemetry and sensor. International Electro-technical Commission (IEC) referred to in the cable category indoor cable, I believe that the Board should include at least the inner fiber optic cabling with fiber optic cable and two parts. Bureau of cloth with a fiber optic cable placed inside a central office or other telecommunications room, laying a relatively fixed position and close order. Integrated wiring cable laying on the user side of the room, mainly used by the user, so the vulnerability should be compared with the Bureau of the cable more stringent consider it.

Optical fibers are dielectric, optical media also can fulfill completely metal-free. Such all-dielectric fiber optic cable will be the best power system communication lines. All dielectric fiber optic cable laying for power poles road there are two structures: the all-dielectric 
self-supporting (ADSS) structure and to the overhead structure for winding line. ADSS fiber optic cable can be used alone because of its placement, adapt to a wide range, it has been widely used in the current transformation of Chinese power transmission system. China has been able to produce a variety of ADSS fiber optic cable to meet the needs of the market. But in the product structure and performance, such as the number ambition cable structure, cable creep and arc resistance properties, etc., to be further improved. ADSS fiber optic cable in the country's recent demand for larger, is a popular product.

\section{Development Direction and Development Trend of Optical Fiber Communication}

First, the optical fiber transmission system is to continue to high-speed, high-capacity direction. Wavelength Division Multiplexing (WDM), frequency division multiplexing (FDM), Time Division Multiplexing (TDM) technology to overcome the problems that limit the rate of single-channel rate limits and electronics upgrades difficult. In particular, wavelength division multiplexing technology and optical technology advances, so that the transmission capacity of an optical fiber is now on every 9 to 12 months to double. $5 \mathrm{~Gb} / \mathrm{s}$ system has been used in the trans-Pacific submarine cable system (TPC-5/6) in. Wavelength Division Multiplexing (WDM) systems are also on the submarine cable system. Alcatel 1999 were $32 \times 10 \mathrm{~Gb} / \mathrm{s}$ all-optical transmission distance of $400 \mathrm{~km}$ transmission experiment, the use of forward error correction technology (FEC), far from the pump pre-and post-optical amplifying optical amplifier and Raman amplification and dispersion management technology. In 2002 Alcatel C-band and L-band were 10.2Tb/s (256×42.7Gb/s) transmission distance is $3 \times 100 \mathrm{~km}$ test.

Second, an optical communication system is extensive use of optical technology. With integrated communication rate exceeds the rate limit, in order to increase the rate, the only way out of photonic technologies. First, the optical amplifier (EDFA) applications, can amplify the optical signal directly, replacing the light - electricity - light conversion of electrical relays, it is considered to be a revolution in telecommunications technology. Second, the optical time division multiplexing (OTDM) optical soliton communication technology and the use of non-linear effect of dispersion compensating fiber for ultra high-speed $\mathrm{Tb} / \mathrm{s}$ rate communication opened up a bright prospect. Optical soliton communication although not currently commercially available, but the experiment has done relatively well, has received $160 \mathrm{~Gb} / \mathrm{s}$ data transmission speed record of $200 \mathrm{~km}$. Again, storage, processing and exchange of light signals have become a hot research topic.

Third is to establish fiber-optic broadband integrated services network. With the reduced cost of optical fiber, optical fiber communication applications extend from the trunk to the LAN communications, network users. Fiber to the curb (FTTC), fiber to the building (FTTB), fiber to the user (FTTH) is gradually becoming a reality. SDH transmission system from substituted PDH, increasingly complex network structure, which provides broadband services, distribution network and service management becomes more important. Fourth, the development of optical integration technology has new devices and new technology emerging. In order to meet $\mathrm{Gb} / \mathrm{s}$ applications must implement component-based optoelectronic devices, integrated. Such as lasers and electric drive has been integrated on a single chip, a light detector and preamplifier stage is also electrically integrated on a single chip. Development of high-speed light sources (DBF-LD, MQW-LD) and new fiber also continuously made new progress.

Today, optical fiber communication technology has developed for nearly 40 years and its main part is transmission. But with the increasingly close combination between communication technology with computer technology, coupled with the needs of various optical network scheduling, networking, survivability, control, etc., you need to integrate into a more complete connection control technology in optical networks, automatic discovery protection and recovery capability, ie intelligent optical network optical fiber communication technology as an important development objectives, which is also the future development trend of optical fiber communication technology lies. 


\section{Conclusion}

With greatly enhance the information age of the twenty-first century information needs, optical fiber communication technology with its various advantages become the subject of a communication transmission medium, and gradually penetrate into all areas of society, causing the industry reform. In this era background, only the continuous improvement of optical fiber communication technology, closely work with the pulse of optical fiber communication technology development, can we enhance many aspects of our country's comprehensive national strength and the pace of development of Chinese socialist modernization.

\section{References}

[1] Chesnoy, J, Global, undersea networks: Technical developments, OFC/IOOC 99. Technical Digest, Vol. 1 , 21-26 Feb. 1999 ,Pages:36 - 37.

[2] Pasu Kaewplung; Tuptim Angkacw, Feasibility of 100-Gb/s 10000-kmSingle-Channel Optical Transmission by Midway Optical Phase Conjugation Incorporated with Third-Order Dispersion Compensation, IEEE Photonics Technology Letters, Vol.13,No.4,April 2001.

[3] Alfred W.T. Wu; Arthur J. Lowery, Efficient Multi-wave length Dynamic Model for Erbium-Doped Fiber Amplifier, IEEE Journal of Quantum Electronics, Vol.34, No.8, August 1998.

[4] T YU, W M Reimer, V S Grigoryan, A Mean field approach for simulating wave length-division multiplexed systems, IEEE Photonics Technology Letters, 2000.12(4), 443-445.

[5] Robert I. Killey, Investigation of nonlinear distortion in 40Gb/s transmission with higher order mode fiber dispersion compensators Journal of light wave technology vol 20, No. 12, Dec.2002, 2282-2285. 